\title{
Fluid Vesicles in Shear Flow
}

\author{
Martin Kraus, Wolfgang Wintz, Udo Seifert, and Reinhard Lipowsky \\ Max-Planck-Institut für Kolloid- und Grenzflächenforschung, Kantstrasse 55, 14513 Teltow-Seehof, Germany
}

(Received 10 May 1996)

The shape dynamics of fluid vesicles is governed by the coupling of the flow within the twodimensional membrane to the hydrodynamics of the surrounding bulk fluid. We present a numerical scheme which is capable of solving this flow problem for arbitrarily shaped vesicles using the Oseen tensor formalism. For the particular problem of simple shear flow, stationary shapes are found for a large range of parameters. The dependence of the orientation of the vesicle and the membrane velocity on shear rate and vesicle volume can be understood from a simplified model. [S0031-9007(96)01470-6]

PACS numbers: 87.45.-k, 47.55.Dz, 68.10.-m

Vesicles are closed lipid membranes suspended in aqueous solution. If the composition of this solution inside and outside the vesicle is identical, it affects the equilibrium properties of the vesicle only by osmotically fixing the enclosed volume. Minimizing the bending energy of the membrane under the constraints of fixed enclosed volume and membrane area then yields the equilibrium vesicle shape at rest [1]. The dynamics of this shape, however, is governed by the coupling of the flow within the two-dimensional incompressible fluid membrane to the hydrodynamics of the bulk fluid. Any theory of vesicle dynamics is complicated by the fact that the boundary conditions for the three-dimensional NavierStokes equations have to be evaluated at the vesicle surface, which is moving with the fluid and whose shape is not known a priori.

Considerable practical interest in this problem arises from a more complex system, the red blood cell. The deformation of erythrocytes in hydrodynamic flow fields is used as a measure of changes in the elastic properties of pathologically modified cells $[2,3]$. The understanding of the dynamics of a single cell is also a prerequisite for the understanding of the rheology of blood. Since the erythrocyte has a complicated structure consisting of a fluid lipid/protein bilayer and the spectrin network, theoretical analysis has employed expansions for small flow rate [4] or has focused on simpler model systems such as shells with stretching elasticity and liquid drops with homogeneous surface tension [5-8]. The dynamics of fluid membranes with fixed area and bending rigidity has only been studied in the quasiplanar [9], quasispherical [10], and cylindrical [11] geometry without external flow fields. Further analytical work includes the covariant treatment of the membrane incompressibility [12] and the dynamic renormalization of material parameters [13] in equilibrium. The problem for a closed vesicle with arbitrary shape and external flow is investigated in this Letter for the first time.

For specifity, we investigate a linear shear flow $v_{x}^{0}(\mathbf{r})=$ $\dot{\gamma} z$ with shear rate $\dot{\gamma}$, as sketched in Fig. 1(a), which can be easily realized experimentally [2]. The stationary state of a vesicle in such a flow resulting from our computation is shown in Fig. 1(b). It is characterized by both a finite inclination angle $\theta$ between the longest axis of inertia of the vesicle and the flow direction, and a "tanktreading" tangential motion of the membrane around the vesicle with revolution frequency $\omega$. Both $\theta$ and $\omega$ are found to depend strongly on the reduced volume $v=V /\left(4 \pi R_{0}^{3} / 3\right)$ of the vesicle, where $V$ is the enclosed volume and the surface area $A$ determines the length scale
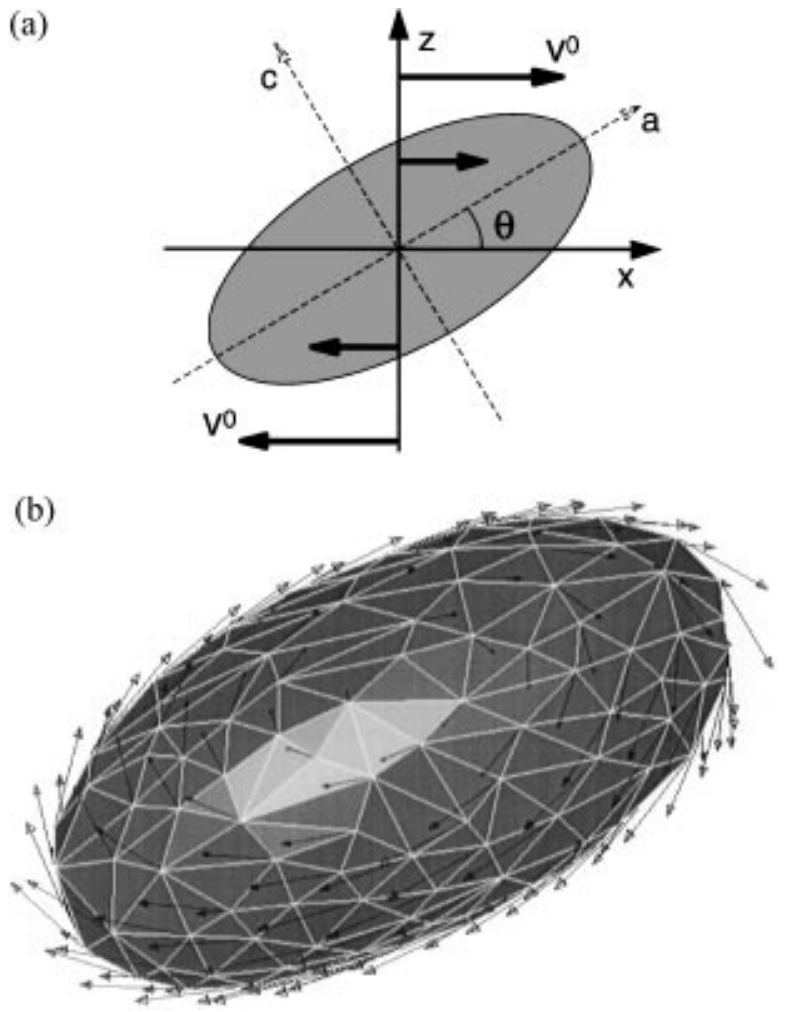

FIG. 1. Vesicle in shear flow $v_{x}^{0}=\dot{\gamma} z$. (a) Schematic drawing of the undisturbed flow, inclination angle $\theta$, and coordinate axes $x, z, a$, and $c$. The coordinate axis pointing into the paper plane is $y$. (b) Stationary state obtained by numerical integration for $v=0.9, \dot{\gamma}=1 \mathrm{~s}^{-1}, \kappa=10^{-19} \mathrm{~J}, \eta=10^{-3} \mathrm{Js} / \mathrm{m}^{-3}$, and $R_{0}=10 \mu \mathrm{m}$. The velocities at the vertices are shown as arrows with length proportional to velocity. 
$R_{0}=\sqrt{A / 4 \pi}$. The bending rigidity of the membrane $\kappa$ sets the energy scale [14]. Together with the bulk viscosity $\eta$, these quantities define an intrinsic time scale $\tau \equiv \eta R_{0}^{3} / \kappa$. Thus, the dimensionless shear rate $\chi \equiv$ $\dot{\gamma} \tau=\dot{\gamma} \eta R_{0}^{3} / \kappa$ is the second parameter of the problem. Figures 2 and 3 show the inclination angle $\theta$ and the mean revolution frequency $\bar{\omega}$ [15], respectively, as a function of the reduced volume $v$ for different values of $\chi$.

We find numerically that, for any nonzero shear rate, small perturbations of the equilibrium shape do not allow stationary flow with the appropriate boundary conditions [16]. Thus, shear appears to be a singular perturbation that drives the vesicle towards a stationary nonequilibrium shape that can be distinctly different from the corresponding equilibrium shape. Further increasing the shear rate $\chi$ only marginally affects shape and orientation angle $\theta$. This behavior is remarkably different from the behavior of both erythrocytes, which go through a transition from tumbling to tanktreading behavior with increasing $\dot{\gamma}$ [2], and liquid drops, which deform to more and more elongated shapes as a function of $\dot{\gamma}$ until they break up [17].

The stationary state was found to be independent of initial conditions. In particular, for $v \leqq 0.75$, oblate discocytes are locally stable in the absence of an external flow field [18] but still transform to the same elongated shape as prolate vesicles when suspended in shear flow. We have tested this behavior for all $v \geq 0.52$, i.e., the entire range where the equilibrium discocytes do not selfintersect.

Hydrodynamic calculations on typical length and time scales of membranes and vesicles are simplified by employing the Stokes approximation which is valid in the limit of small Reynolds number Re [19]. For a vesicle suspended in a shear flow with typical velocity $u=\dot{\gamma} R_{0}$, this number can be estimated by $\operatorname{Re}=\rho u R_{0} / \eta=\rho \dot{\gamma} R_{0}^{2} / \eta$.

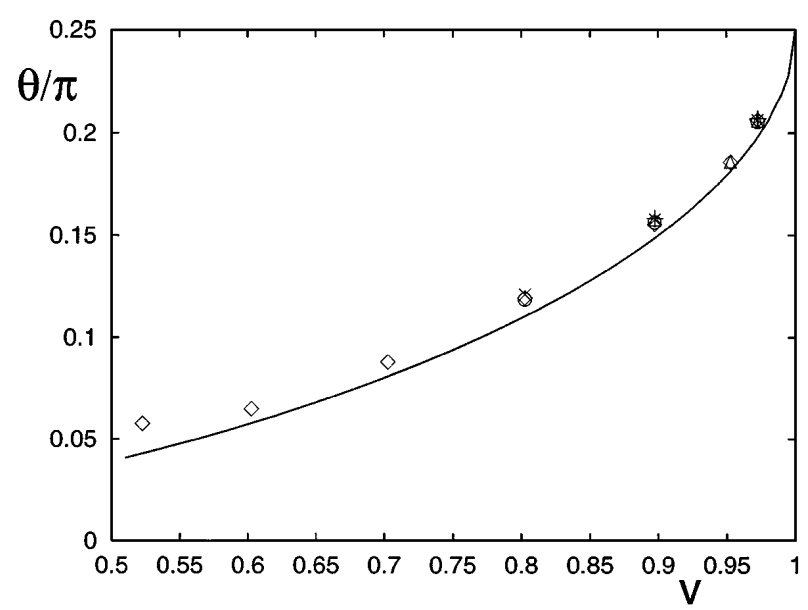

FIG. 2. Inclination angle $\theta$ between the $x$ axis and the longest axis of inertia as a function of reduced volume $v$ for different reduced shear rates $\chi=1(\triangle), 5(\bigcirc), 10(\diamond), 50(\times), 100$ $(+)$. Numerical errors are smaller than the symbol sizes. The continuous line follows from Eq. (8).
Inserting the vesicle size $R_{0} \simeq 10 \mu \mathrm{m}$ and the viscosity $\eta=10^{-3} \mathrm{Js} / \mathrm{m}^{3}$ and density $\rho=10^{3} \mathrm{~kg} / \mathrm{m}^{3}$ of water, the limit of small Re corresponds to $\dot{\gamma} \ll 10^{4} \mathrm{~s}^{-1}$. Thus, the hydrodynamic equations for the velocity field $\mathbf{v}(\mathbf{r})$ of an incompressible Newtonian bulk fluid with external force density $\mathbf{k}(\mathbf{r})$ reduce to the instantaneous force balance

$$
\eta \nabla^{2} \mathbf{v}=\nabla p(\mathbf{r})+\mathbf{k}(\mathbf{r}) .
$$

The pressure field $p(\mathbf{r})$ can be eliminated by using the incompressibility condition $\nabla \cdot \mathbf{v}(\mathbf{r})=0$. Because of the linearity of (1), $\mathbf{v}(\mathbf{r})$ can be written as a superposition of a background flow $\mathbf{v}^{0}(\mathbf{r})$ and additional flows $\mathbf{v}^{i}(\mathbf{r})$ arising from the interactions of the fluid with the membrane.

We describe the membrane surface by a triangulation with vertex positions $\mathbf{R}^{\alpha}$ moving with the adjacent bulk fluid due to non-slip boundary conditions and the impermeability of the membrane for bulk flow. Forces on the membrane are computed on these vertices from the energy

$$
\mathcal{G}\left(\left\{\mathbf{R}^{\alpha}\right\}\right)=\sum_{\alpha} 2 \kappa\left(H^{2}\right)^{\alpha}+\sum_{\alpha} \sigma^{\alpha} A^{\alpha}
$$

of the discretized membrane. The energy has two contributions. First, the discretized squared mean curvature $\left(H^{2}\right)^{\alpha}$ [20] describes the bending energy [14,21,22] with bending rigidity $\kappa$. Second, a locally varying isotropic tension $\sigma^{\alpha}$ which is conjugate to the area $A^{\alpha}$ of the neighboring triangles around each vertex is needed to ensure local incompressibility of the membrane.

The force $\mathbf{K}^{\beta}$ at vertex $\mathbf{R}^{\beta}$ then reads

$$
\begin{aligned}
\mathbf{K}^{\beta} & \equiv-\frac{\partial \mathcal{G}\left(\left\{\mathbf{R}^{\alpha}\right\}\right)}{\partial \mathbf{R}^{\beta}} \\
& =-2 \kappa \sum_{\alpha} \frac{\partial\left(H^{2}\right)^{\alpha}}{\partial \mathbf{R}^{\beta}}-\sum_{\alpha} \sigma^{\alpha} \frac{\partial A^{\alpha}}{\partial \mathbf{R}^{\beta}},
\end{aligned}
$$

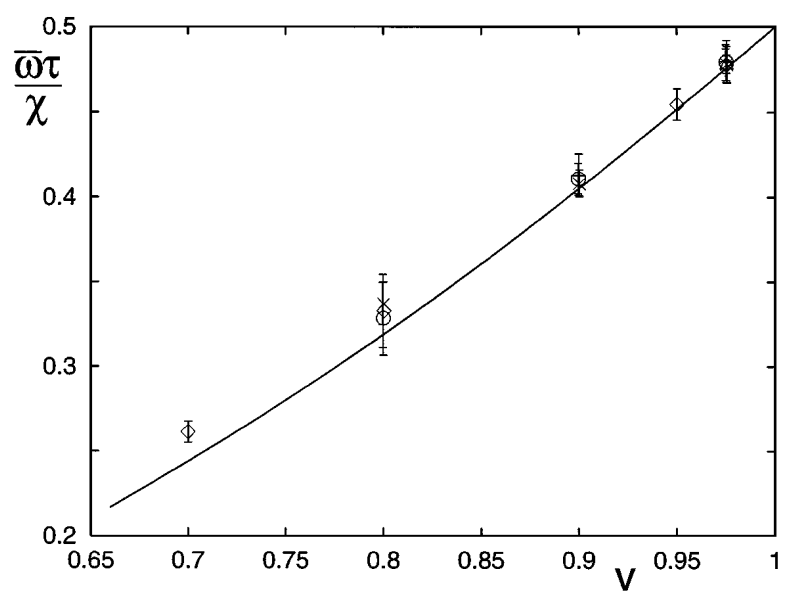

FIG. 3. Scaled revolution frequency $\bar{\omega} \tau / \chi$ averaged over all vertices as a function of reduced volume $v$ for different reduced shear rates $\chi$ (Symbols are as in Fig. 2). The continuous line is computed using the solution of (9). For volumes $v<0.7$, a full revolution in the stationary state could not be computed due to constraints on computation time. 
and the force density is given by $\mathbf{k}(\mathbf{r})=\sum_{\beta} \delta(\mathbf{r}-$ $\left.\mathbf{R}^{\beta}\right) \mathbf{K}^{\beta}$.

The disturbance flow due to the presence of the vesicle can be computed using the Oseen tensor $\mathbf{O}$ describing the flow field induced by a discrete point force $\mathbf{K}^{\beta}$ in an infinite fluid medium. The total velocity at the vertex position $\mathbf{R}^{\alpha}$ then reads

$$
\mathbf{v}\left(\mathbf{R}^{\alpha}\right)=\mathbf{v}^{0}\left(\mathbf{R}^{\alpha}\right)+\sum_{\beta} \mathbf{O}\left(\mathbf{R}^{\alpha}, \mathbf{R}^{\beta}\right) \mathbf{K}^{\beta} .
$$

The matrix elements of the Oseen tensor are [23-25]

$$
\mathbf{O}_{i j}\left(\mathbf{R}^{\alpha}, \mathbf{R}^{\beta}\right)=\frac{1}{8 \pi \eta d}\left(\delta_{i j}+\frac{d_{i} d_{j}}{d^{2}}\right),
$$

with $\mathbf{d} \equiv \mathbf{R}^{\alpha}-\mathbf{R}^{\beta}$ and $d \equiv|\mathbf{d}|$. Here, the viscosity of the fluid $\eta$ is assumed to be equal for the fluids inside and outside the vesicle [26].

After dealing with the energetics of the membrane in the force balance we have to treat the dynamics of the lipid membrane correctly. Two-dimensional incompressibility of the membrane leads to the condition that the area around every vertex remains constant under the dynamics, i.e., $0=\partial_{t} A^{\alpha}=\sum_{\beta}\left(\partial A^{\alpha} / \partial \mathbf{R}^{\beta}\right) \mathbf{v}\left(\mathbf{R}^{\beta}\right)$. Using Eq. (4) for the velocities and Eq. (3) for the vertex forces, this condition yields an inhomogeneous system of linear equations for the local tensions $\sigma^{\alpha}$. After the tensions are thus determined, moving the vertices by $\Delta t \mathbf{v}\left(\mathbf{r}^{\alpha}\right)$ with a short time step $\Delta t$ respects the Stokes equations and all boundary conditions and thus gives a correct integration scheme for the hydrodynamic problem which automatically conserves the volume of the enclosed fluid. Fluidity of the membrane is ensured by bond flips attempted at regular intervals [27].

This procedure turns out to be stable in the sense that (i) an initially smooth surface remains smooth during time evolution, and (ii) the distribution of triangle sizes and angles remains approximately constant in the stationary state. As a test, we relax surfaces at $\mathbf{v}^{0}=0$ and arrive at the known axisymmetric equilibrium shapes [1] with an error of $0.2 \%$ for the energy and $1.5 \%$ for the (uniform) tension using a discretization with $N=337$ vertices. Area and volume remain constant with an error below $0.1 \%$ for the longest runs.

The numerical results for shape, orientation, and membrane velocity in the stationary state may be understood using a simplified model. The numerical data show an approximate mirror symmetry of the induced tension $\sigma$ with respect to the plane defined by the axis denoted by $a$ in Fig. 1(a) and the $y$ axis. The axis perpendicular to this symmetry plane is denoted by $c$. We now assume that this symmetry holds exactly. The velocity field can be written as a superposition of the undisturbed shear flow $\mathbf{v}^{0}$ and the flow $\mathbf{v}^{i}$ induced by all forces on the membrane. The latter flow must have the mirror symmetry of the inducing forces. In a stationary state, the sum of these two contributions has to be tangential to the membrane surface. We evaluate this condition for the contour of the vesicle in the $x-z$ plane at $y=0$. With $s$ denoting the arclength of this contour, the contour is described by a vector $(a(s), 0, c(s))$ with the local tangential velocity $\beta(s)$. We obtain four equations,

$$
\begin{aligned}
& \pm \beta(s) \frac{d a(s)}{d s}=v_{a}^{i}+\dot{\gamma} \cos \theta[a(s) \sin \theta \pm c(s) \cos \theta], \\
& \beta(s) \frac{d c(s)}{d s}= \pm v_{c}^{i}-\dot{\gamma} \sin \theta[a(s) \sin \theta \pm c(s) \cos \theta],
\end{aligned}
$$

where the different signs apply to the upper and to the reflected point on the lower part of the vesicle, respectively.

We can now eliminate the unknown quantity $\mathbf{v}^{i}$ from (6) and (7) and obtain differential equations for $d a(s) / d s$ and $d c(s) / d s$. These equations describe an ellipse

$$
\frac{a^{2}(s)}{r^{2} \cos ^{2} \theta}+\frac{c^{2}(s)}{r^{2} \sin ^{2} \theta}=1 .
$$

If one now assumes axisymmetry of the entire shape, the length scale $r$ and the angle $\theta$ are uniquely determined by the constraints on area and volume of the vesicle. The shear rate $\dot{\gamma}$ scales the velocity $\beta(s)$ and does not influence the shape or orientation. We obtain the differential equation [28]

$$
\frac{d \beta(s)}{d s}= \pm \frac{\dot{\gamma} a(s) c(s) \sin ^{2} \theta \cos ^{2} \theta\left(\cos ^{2} \theta-\sin ^{2} \theta\right)}{a^{2}(s) \sin ^{4} \theta+c^{2}(s) \cos ^{4} \theta}
$$

for the velocity along the contour by forming the derivative of the geometrical condition $[d a(s) / d s]^{2}+[d c(s) / d s]^{2}=1$ with respect to $s$. This equation can be solved numerically, giving the revolution frequency $\omega \equiv 2 \pi / \oint \beta(s)^{-1} d s$.

In the spherical limit $v \approx 1$, Eq. (8) gives $\theta \approx \pi / 4$ and $r \approx R_{0}$. Equation (9) simplifies to $\beta(s)=\dot{\gamma} R_{0} / 2=$ const, which is equivalent to $\omega=\dot{\gamma} / 2=\chi / 2 \tau$. Thus, all limit values for a spherical vesicle are identical to the results for a rigid sphere or a fluid drop with infinite surface tension in shear flow [19].

As Figs. 2 and 3 show, our simplified model yields good quantitative agreement with the results of the hydrodynamic integration. The results for different shear rate $\chi$ collapse as expected. The spherical limit $v \approx 1$ cannot be reached numerically, as the tension in the membrane diverges when exterior forces are applied to a sphere. For reduced volumes $v \leq 0.8$, the symmetry assumptions in our simplified model are less justified.

The results of the numerical integration contain information about the distribution of velocity and tension within the membrane not available from the simplified model. The revolution frequency $\bar{\omega}$ measures only the mean velocity. The local variations of membrane velocity show two remarkable features: (i) The velocity varies along the contour in a way that velocities are smaller towards the poles of the vesicle. This behavior can be 


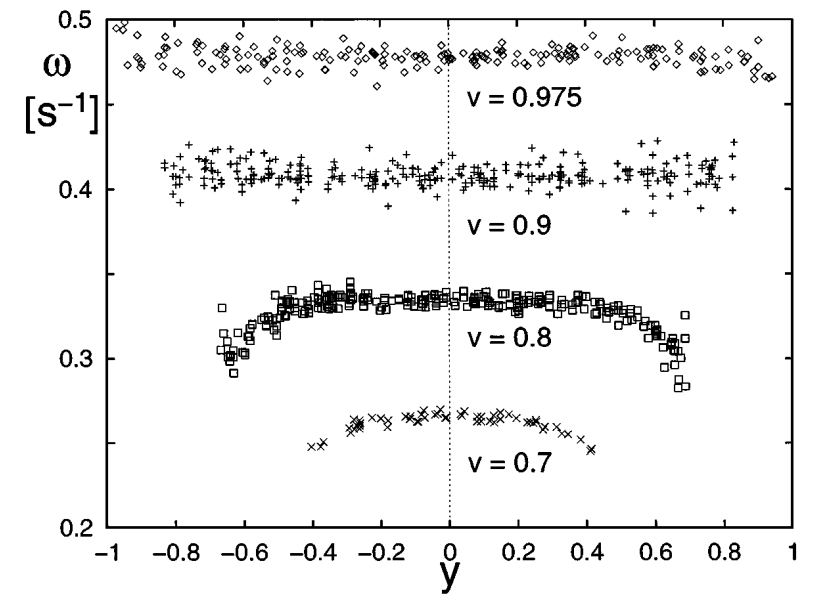

FIG. 4. Revolution frequency $\omega$ as a function of lateral coordinate $y$ for reduced volumes $v=0.7,0.8,0.9,0.975$ for $\chi=10, \tau=10 \mathrm{~s}$. The $y$ independence of $\omega$ for large $v$ implies that adjacent membrane segments are again close together after a full revolution.

understood qualitatively by formulating (6) and (7) for a contour rotated out of the $x-z$ plane. (ii) Figure 4 shows the lateral $(y)$ variation in the revolution frequencies $\omega$ evaluated for individual vertices. Even though we have included neither an explicit shear rigidity nor shear viscosity, we find numerically effective shear in the membrane only for large deviations from an elliptical shape at small $v$.

In the stationary state, the membrane tension $\sigma^{\alpha}$ is constant only for $\mathbf{v}^{0}=0$. In a shear flow, we find that the largest values of the (negative) tension are in the middle of the vesicle, i.e., the vesicle responds to being pulled apart by the shear flow. For large shear rates $\chi \gg$ 1 , bending rigidity is irrelevant, and the mean tension $N^{-1} \sum_{\alpha} \sigma^{\alpha}$ increases linearly with $\chi$, thus dominating the elastic energy of the membrane.

In conclusion, we have developed a stable numerical scheme for calculating the time evolution of vesicles with arbitrary shape suspended in arbitrary flow fields. In the case of simple shear flow, we find an inclination angle $\theta$ independent of shear rate and a revolution frequency $\omega$ linearly proportional to shear rate. Our approach breaks down for shear rates smaller than $\chi \approx k_{B} T / \kappa \simeq 0.04$, where typical velocities of rotational diffusion $k_{B} T / \eta R_{0}^{2}$ are comparable to the velocity of the shear flow. In this regime, one should incorporate thermal fluctuations.

We thank T. M. Fischer for helpful discussions. M. K. and W. W. thank K. Kehr from IFF, Forschungszentrum Jülich for hospitality. U.S. benefited from interaction with S. Langer and M. Wortis on a related problem.

[1] U. Seifert and R. Lipowsky, in Structure and Dynamics of Membranes, edited by R. Lipowsky and E. Sackmann (Elsevier Science, Amsterdam, 1994).

[2] T.M. Fischer, M. Stöhr-Liesen, and H. SchmidSchönbein, Science 24, 894 (1978).
[3] W. Groner, N. Mohandas, and M. Bessis, Clin. Chem. 26, 1435 (1980).

[4] M. A. Peterson, Phys. Rev. A 45, 4116 (1992).

[5] S. R. Keller and R. Skalak, J. Fluid Mech. 120, 27 (1982).

[6] D. Barthès-Biesel, Physica (Amsterdam) 172A, 103 (1991).

[7] W. S. J. Uijttewaal, E. J. Nijhof, and R. M. Heethaar, Phys. Fluids A 5, 819 (1993).

[8] C. Pozrikidis, J. Fluid Mech. 297, 123 (1995).

[9] F. Brochard and J. F. Lennon, J. Phys. (France) 36, 1035 (1975).

[10] S. T. Milner and S. A. Safran, Phys. Rev. A 36, 4371 (1987).

[11] P. Nelson, T. Powers, and U. Seifert, Phys. Rev. Lett. 74, 3384 (1995).

[12] G. Foltin, Phys. Rev. E 49, 5243 (1994).

[13] W. Cai and T. C. Lubensky, Phys. Rev. E 52, 4251 (1995).

[14] We employ the simplest possible curvature model for the membrane bending energy, neglecting spontaneous curvature of the membrane as well as effects of bilayer elasticity [1]. These would introduce additional length scales.

[15] Numerically, we determine the mean tanktreading time $t_{t}=2 \pi / \omega$ as twice the time between two successive sign changes of the coordinate $z$ for a single vertex, and average over all vertices in order to obtain $\bar{\omega}$.

[16] The smallest $\chi$ for which we could obtain numerically stable data is $\chi=0.1$, cf. M. Kraus, Ph.D. thesis, Universität Potsdam, 1996 (unpublished).

[17] J. M. Rallison, Annu. Rev. Fluid Mech. 16, 45 (1984).

[18] M. Kraus, U. Seifert, and R. Lipowsky, Europhys. Lett. 32, 431 (1995).

[19] T. G. M. van de Ven, Colloidal Hydrodynamics (Academic Press, London, 1989).

[20] Squared mean curvature is discretized by calculating the mean curvature on the edges of the triangulation and subsequently summing up and squaring on adjacent vertices.

[21] P. B. Canham, J. Theor. Biol. 26, 61 (1970).

[22] W. Helfrich, Z. Naturforsch. 28C, 693 (1973).

[23] C. Pozrikidis, Boundary Integral and Singularity Methods for Linearized Viscous Flow (Cambridge University Press, Cambridge, England, 1992).

[24] M. Doi and S.F. Edwards, The Theory of Polymer Dynamics (Clarendon Press, Oxford, 1986).

[25] The Oseen tensor diverges for $d \longrightarrow 0$. The selfinteraction term $\mathbf{O}^{\mathrm{f}}\left(\mathbf{R}^{\alpha}, \mathbf{R}^{\alpha}\right)$ can be regularized by integrating over the adjacent triangles of $\mathbf{R}^{\alpha}$, cf. Ref. [7]. As this integration procedure is employed only for this special case and not for the interaction between arbitrary vertices, we have to introduce a weighting factor $c^{\alpha}$ for the self-interaction which is chosen to ensure constancy of the vesicle volume. Usually, we find $\max _{\alpha}\left|1-c^{\alpha}\right|<0.05$.

[26] Different viscosities inside and outside the vesicle membrane lead to an integral equation in $\mathbf{v}$ for every time step, cf. Ref. [23].

[27] D. M. Kroll and G. Gompper, Science 255, 968 (1992), and references therein.

[28] Positive sign applies to $a c<0$, and negative sign to $a c>0$. 